\title{
Improvement of reproducibility of macular volume measurements using the Heidelberg retinal tomograph
}

\author{
A Ang, L Tong, S A Vernon
}

\begin{abstract}
Aims-To develop a more reproducible method of macular volumetric analysis in order to facilitate serial monitoring of changes in retinal oedema with time.

Methods-The Heidelberg retinal tomography (HRT) machine was used to scan the macula of 20 normal subjects and 40 diabetics with macular oedema. The volume above reference plane (VARP) within a $2 \mathrm{~mm}$ diameter and $3 \mathrm{~mm}$ diameter circle was measured twice in each eye. The position of the circle to be measured was carefully defined relative to major retinal vessels. As a modification to the previously published technique, fluctuation of the height of the contour line relative to the focal plane of the eye is compensated for by an adjustment of the reference plane. The position of the circle was controlled relative to major retinal vessels using an acetate. The reproducibility of VARP measurement was assessed by three variables-the standard error of the difference on two isolated VARP measurements, the standard error of the difference between the average of three readings on two different occasions, as well as the coefficient of variation (COV).
\end{abstract}

Results-Both the $2 \mathrm{~mm}$ and $3 \mathrm{~mm}$ circles showed good reproducibility in VARP measurements. In normal subjects, the COV of VARP for $2 \mathrm{~mm}$ circles and $3 \mathrm{~mm}$ circles were $13 \%$ and $12 \%$ respectively. In patients with macular oedema, the COV of VARP for $2 \mathrm{~mm}$ circles and $3 \mathrm{~mm}$ circles were $9 \%$ and $11 \%$ respectively.

Conclusion-The modification of VARP measurements between scans of the same eye has improved the COV from $31 \%$ to $9 \%$ in eyes with diabetic macular oedema. (Br f Ophthalmol 2000;84:1194-1197)

The assessment of changes in the retina is an integral part of the management of posterior segment diseases. Difficulties arise when relatively small volumes of retina are involved in the disease process, and diabetic macular oedema is the paradigm of such a clinical scenario.
There is a need for a simple, accurate, non-invasive, and practical way to follow patients who may develop changes in central retinal volume. A number of researchers, including ourselves, have attempted to measure either retinal thickness or retinal volume values. $^{1-6}$ Our initial method of volume measurement resulted in rather high coefficient of variation (COV) results in diabetics with macular oedema. ${ }^{6}$

As a result of this, we have developed a modification to our original technique of measuring retinal volumes using the Heidelberg retinal tomograph (HRT) in an attempt to improve the reproducibility found in our initial methodology. ${ }^{6}$

The HRT is a scanning laser ophthalmoscope with confocal optics. It takes 32 images of the fundus in 1.6 seconds in a plane perpendicular to the optical axis. The computer software then aligns the images so as to eliminate the effect of eye movement and compiles a three dimensional image of the surface scanned. This instrument has originally been used for the analysis of optic nerve head topography but it has recently been adapted for macular investigations. ${ }^{2356}$

\section{Method}

Forty eyes of diabetic patients with macular oedema (20 with previous focal laser photocoagulation, 20 without) and 20 eyes of normal subjects were scanned using the HRT.

Macular oedema was defined as retinal thickening evident on contact lens examination or slit lamp biomicroscopy with a 78D lens within 2 disc diametres from the centre of the fovea. Normal subjects were volunteers who had no fundal abnormalities clinically. Cycloplegia was achieved before scanning with topical cyclopentolate $1 \%$, and phenylephrine $2.5 \%$ was used to achieve adequate dilatation in all subjects. The refractive error of the patients ranged from -6 to +6 dioptres. The study was performed with approval of the ethics committee of Queen's Medical Centre. Informed consent was obtained from each participant before HRT scanning.

The scans were performed by an experienced operator (LMT). The scanning technique has been described in detail in other reports. ${ }^{56}$ To assess the repeatability of volume
Accepted for publication 26 April 2000 


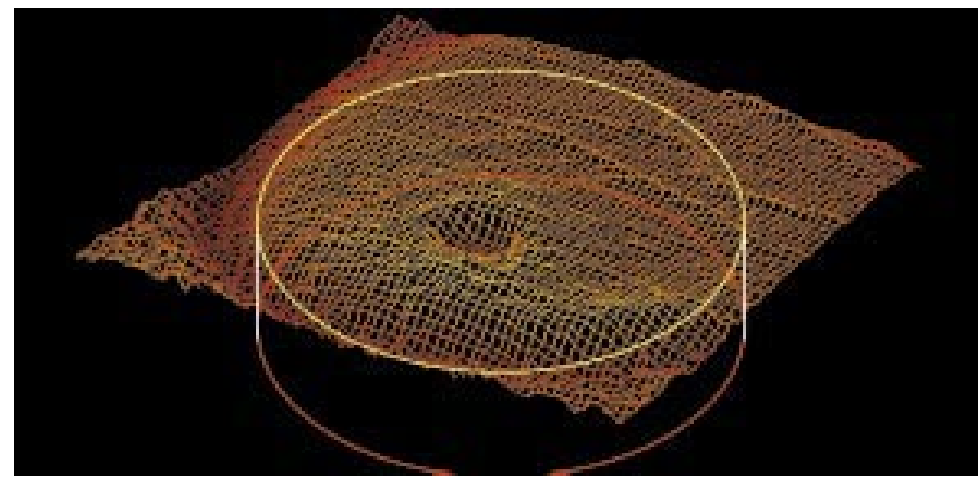

Figure 1 (Not to scale) The volume above reference plane (VARP) is a "cylindrical" space bounded by: (a) the retinahyaloid surface anteriorly, (b) the cylindrical body with radius 1 or $1.5 \mathrm{~mm}$, and (c) a circular surface of the same radius posteriorly (the reference plane).

measurements, two scans of each eye were obtained on the same day. In between the scans, the patient was asked to sit back and the head repositioned to simulate as closely as possible a separate examination. It would be inappropriate to scan on different days in case real retinal volumes varied.

The first scan was taken as the baseline scan. A $2 \mathrm{~mm}$ diameter contour circle centred on the fovea was drawn on the scan. A reference plane below the contour line was selected so that no retina within the circle would lie below this plane. This was achieved by ensuring that the volume below the reference plane was just zero at the HRT computation.

The volume of the retina above the reference plane (VARP) bounded by the contour circle was then determined by the HRT Software 2.01 (Fig 1). The mean height of the contour circle along the $\mathrm{z}$ axis was noted. A transparency was then placed on the computer monitor, the major blood vessels traced, and the centre of the contour circle marked.

For subsequent scans, the same transparency was used to determine the centre of the contour circle, which was then drawn using the "circle draw" facility of the software. The reference plane of the second scan was imported from the first to keep it at a same depth below the mean height of the new contour line (see Fig 2). The VARP measurement of the second scan was calculated by the software for comparison with that of the first scan. The process was then repeated for a 3 $\mathrm{mm}$ diameter circle.

\section{DATA ANALYSIS}

Normal subjects

For the pairs of VARP measurements, the data analyses were performed according to previously published methodology. ${ }^{3}$ Briefly, the variance of each pair of VARP readings was obtained and a pooled standard deviation obtained for the group of subjects. Using formulas from Menezes et $a l{ }^{3}$ the pooled standard deviation (S) was then used to calculate the $95 \%$ confidence interval of the standard error of the difference (1) between two single readings in any normal subject $\left(\mathrm{SE}_{1}\right)$ and also (2) between the average of three VARP readings in three scans on two occasions $\left(\mathrm{SE}_{3}\right)$. The mean $(\mathrm{M})$ and standard deviation (SD) of the VARP values were determined.

The coefficient of variation (COV) was also calculated, in order to facilitate comparison with our previous method. ${ }^{6}$

\section{Patients with diabetic macular oedema}

The above calculations were performed to determine the $\mathrm{M}, \mathrm{SD}, \mathrm{S}, \mathrm{SE}_{1}$, and $\mathrm{SE}_{3}$ for the two subgroups (with or without laser) and also for the group as a whole. The COVs were also calculated. To determine if there was any difference in the reproducibility between the groups with and without laser, the variances of the VARPs were compared using the unpaired two tailed $t$ test at the significance level of $\mathrm{p}=0.05$.

\section{Results}

Table 1 shows the $\mathrm{M}, \mathrm{SD}, \mathrm{S}, \mathrm{SE}_{1}, \mathrm{SE}_{3}$, and $\mathrm{COV}$ in the various groups. Both the $2 \mathrm{~mm}$ and $3 \mathrm{~mm}$ circles showed good reproducibility in VARP measurements. The $\mathrm{S}, \mathrm{SE}_{1}$, and $\mathrm{SE}_{3}$ can be seen to be small compared with the magnitude of the VARPs used in this study.

There was no significant difference in the reproducibility of VARP measurements be-
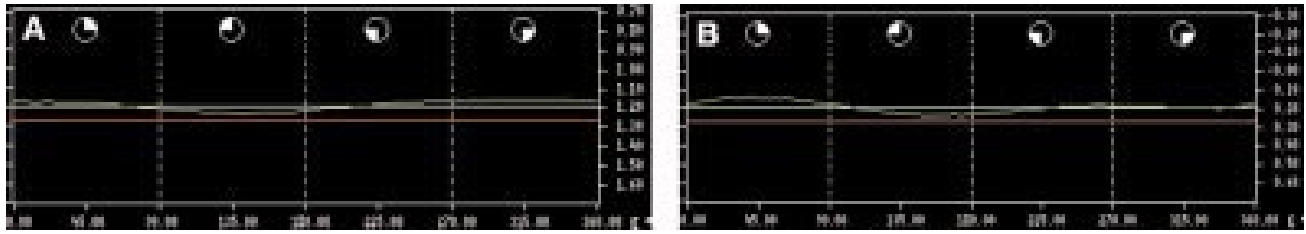

Figure 2 Contour line profile in a diabetic patient's HRT scans. Green line $=$ contour circle profile; red line $=$ reference plane. In the baseline scan (A), the mean height of the contour circle is $1.184 \mathrm{~mm}$. The reference plane is placed at 1.260 $\mathrm{mm}$. In the follow up scan (B), the mean height of the contour circle is $0.189 \mathrm{~mm}$. In order to measure the VARP in the second scan, the reference plane is repositioned at $0.265 \mathrm{~mm}(1.260+(0.189-1.184))$.

Table $1 M$ and $S D$ of the VARP values plus the $S, S E_{1}, S E_{3}$, and COV of the VARP differences

\begin{tabular}{|c|c|c|c|c|c|c|c|c|}
\hline \multirow[b]{2}{*}{ Normal macula } & & & \multicolumn{6}{|l|}{ Macular oedema } \\
\hline & & & No laser & & With laser & & Combined & \\
\hline Diameter of circle (mm) & 2 & 3 & 2 & 3 & 2 & 3 & 2 & 3 \\
\hline$M\left(\mathrm{~mm}^{3}\right)(\mathrm{SD})$ & $0.145(0.075)$ & $0.426(0.247)$ & $0.270(0.113)$ & $0.598(0.283)$ & $0.367(0.211)$ & $0.852(0.434)$ & $0.319(0.174)$ & $0.715(0.375)$ \\
\hline $\mathrm{S}\left(\mathrm{mm}^{3}\right)$ & 0.019 & 0.046 & 0.022 & 0.043 & 0.046 & 0.082 & 0.036 & 0.065 \\
\hline $\mathrm{SE}_{1}\left(\mathrm{~mm}^{3}\right)$ & 0.054 & 0.132 & 0.060 & 0.120 & 0.129 & 0.231 & 0.101 & 0.185 \\
\hline $\mathrm{SE}_{3}\left(\mathrm{~mm}^{3}\right)$ & 0.031 & 0.036 & 0.036 & 0.071 & 0.075 & 0.133 & 0.058 & 0.107 \\
\hline $\operatorname{COV}(\%)$ & 13 & 12 & 8 & 7 & 12 & 9 & 9 & 11 \\
\hline
\end{tabular}


tween the diabetic maculae with previous laser and those without $(\mathrm{p}>0.05)$.

\section{Discussion}

The HRT was initially designed for optic disc topography. Our group has described a method for obtaining volumetric measurements of the macula using the HRT. ${ }^{56}$ Its effectiveness in the detection of sight threatening diabetic maculopathy in conjunction with its other facilities for imaging the macula has been evaluated. ${ }^{7}$ In this study, we have described a new method designed to improve the reproducibility of repeated (same day) macular volume measurements which will facilitate monitoring of volumetric changes in macular disorders. Zambarakji et al attained a coefficient of variation of $20 \%$ in normal maculas ${ }^{5}$ and $31 \%{ }^{6}$ in maculas with oedema. This was obtained using a slightly different method of ascertainment of VARP. In the present study the COV of VARP has been calculated to be $7-13 \%$ (see Table 1 ).

One of the factors in the improved repeatability is likely to be the method of relocalisation of the contour circle for interscan comparisons. This has been achieved by identification of the position of the centre of the contour circle with respect to the major retinal vessels which are presumed not to vary between scans.

The HRT computer software (version 2.01) can import a contour circle from a baseline scan to a follow up scan but the algorithm of this is not stated. Although not formally measured we have noted that the position of an imported circle on the macula can disagree with the position of circles defined by a transparency. In our previous study, circles were redrawn on scans without the control of tracings on transparencies. ${ }^{6}$

Another major factor involved the adjustment of the reference plane between scans. Menezes et $a l^{\beta}$ have reported the reproducibility of measuring the mean height of points along the perimeter of a circle of radius 0.615 $\mathrm{mm}$ centred on the fovea, using the HRT. They found that variability of the mean height readings arise from the variation of the position of the contour surface from the focal plane of the eye along the $\mathrm{z}$ axis. In other words the focal plane of the eye as determined by the HRT machine may not be at a constant distance from the retinal surface. Menezes et al also reported the reproducibility of mean depth readings in the same paper. This referred to the difference between the mean height values of points within the circle compared with points along the perimeter of the circle. This parameter has a smaller standard deviation or variability compared with the mean height, as the subtraction involved in the computation would eliminate variability in the position of the focal plane.

It follows from the above that to make meaningful comparisons between VARP readings in different scans, it would be necessary to keep the reference plane at a constant distance from the contour line.
Other methods of HRT macular measurement have been reported. ${ }^{23}$ Analysis of the normalised mean $\mathrm{z}$ profile signal width was done by Hudson et al. ${ }^{2}$ Repeatability was $20 \%$ of the measured value but it involved seven topographic images for each examination.

The optical coherence tomography $(\text { OCT })^{1}$ and retinal thickness analyser (RTA) ${ }^{4}$ can be used to measure retinal thickness. In the OCT, ${ }^{1}$ accurate localisation of the area of interest on the retina is difficult because it depends on the ability of the patient to fixate on a fixation light. The image acquisition requires patients to keep the eye immobile for 2.6 seconds. As the final image is a two dimensional cross sectional image its position with respect to landmarks on the retina surface is uncertain.

Zeimer et $a l^{4}$ described a method of "topographic" analysis of the macula using the scanning RTA, but in order to study a $6 \mathrm{~mm}$ by 6 $\mathrm{mm}$ area of the macula, nine scans of the eye with patient fixing nine times must be combined for interpretation. They reported the coefficient of variability of the retinal thickness values of corresponding points on three scans on the same day to be $3.6 \%$. The raw values were, however, in arbitrary units, and conversion to micrometres required measurement of the axial length of the eye by A-scan ultrasonography and entering this into an optical model of the eye.

It must be pointed out that the volume measured (VARP) is not the absolute anatomical volume of the retina in the area concerned but rather an arbitrary value to allow comparison with future measurement.

Increased sensitivity, theoretically, can be achieved by using the average of three scans on each examination visit (compare SE1 with SE3 in Table 1).

Macular changes "beyond what is expected from measurement error" can be detected on two occasions using the HRT - that is, by comparing the magnitude of the difference of the measurement of VARP to the values of $\mathrm{SE}_{1}$ or $\mathrm{SE}_{3}$ whichever is appropriate.

For example (from Table 1), if the baseline mean VARP of three scans of a patient with macular oedema (without laser) using the 2 $\mathrm{mm}$ contour circle was $0.270 \mathrm{~mm}^{3}$ then a follow up scan with a VARP that is $0.036 \mathrm{~mm}^{3}$ greater or smaller than $0.270 \mathrm{~mm}^{3}$ would represent a significant change $(\mathrm{p}<0.05)$ - that is, there is less than $5 \%$ probability of such a measurement difference occurring purely by chance. We note that Menezes and others' report did not include the mean values or any description of the actual magnitude of the two parameters studied. ${ }^{3}$

Although in this study we centred the circle on the fovea, this technique may be used to monitor any localised area of retinal anomaly, as long as the contour circle is drawn through normal retina. We assume that the normal retina will not experience any changes in its topography over time. Hence, any changes in the VARP measurements with time would be wholly attributed to changes within the contour circle. Our proposed method is not 
suitable for monitoring of large areas of diffuse retinal oedema.

In reality, the retina underlying the contour circle may become involved by the disease process - for example, in diabetes. This could then lead to changes in the VARP measurements not due entirely to changes within the circle, reducing its accuracy. Subtle changes involving the contour circle may not be detected by clinical examination of the fundus. Therefore it is important to monitor the shape of the contour circle profile as well, for any significant changes between scans which could signify involvement of the contour circle.

The main application of this technique would be in very early clinically significant diabetic maculopathy, especially in detecting small increases in macular volume that may not be detected on clinical examination. This would allow earlier intervention, if indicated, before visual acuity in the eye is affected. Following treatment of the maculopathy, the HRT could then be employed to monitor the effects of the treatment. In exudative age related macular degeneration, this technique could be used as an adjunct to fundus fluorescein angiography and indocyanine angiography in the assessment of the natural progression of the disease and also of the effectiveness of the various treatment options by providing a volumetric measurement of the affected area. There are numerous other potential areas of application eg macular hole, choroidal naevus, etc.

Further work remains to be done. Prospective, longitudinal studies would need to be performed to assess the usefulness of this technique in clinical practice. This is hampered by the lack of a gold standard in volumetric measurement with which to compare this technique. Other difficulties include (as mentioned above) the monitoring of the contour circle to ensure that the retina through which it is drawn remains normal. Apart from subjectively as- sessing the contour circle profile for significant changes between scans, a more objective method involves calculating the area made between the contour circle profile and the mean contour height line. Localised changes in the contour circle profile with time would be reflected by changes in the calculated area. This function is not currently available in the HRT software version 2.01.

Other potential modifications for future HRT software versions include the incorporation of a function for accurate importation of the contour circle from the baseline scan to a follow up scan, which would make tracing on transparencies unnecessary. Also the formulas for calculation of the reference plane height could be included.

In summary, the above technique is a promising method of obtaining volumetric measurements with possible clinical and research applications, and would appear to represent a significant advance over our former method of volumetric measurement when sequential analysis is required.

Proprietary interests: None

Financial support: None.

1 Hee M, Puliafito C, Wong C. Quantitative assessment of macular edema with optical coherence tomography. Arch Ophthalmol 1995;113:1019-29.

2 Hudson C, Flanagan J, Turner G, et al. Scanning laser tomography Z profile width as an objective index of macular retinal thickening. Br f Ophthalmol 1998;82:121-30.

3 Menezes A, Giunta M, Chisholm L, et al. Reproducibility of topographic measurements of the macula with a scanning laser ophthalmoscope. Ophthalmology 1995;102:230-5.

4 Zeimer R, Shahidi M, Mori M, et al. A new method for rapid mapping of the retinal thickness at the posterior pole. Invest Ophthalmol Vis Sci 1996;37:1994-2001.

5 Zambarakji H, Evans J, Amoaku W, et al. Reproducibility of volumetric measurements of normal maculae with the Heidelberg retina tomograph. Br f Ophthalmol 1998;82:88491.

6 Zambarakji H, Spencer A, Amoaku W, et al. Reproducibility A A with the Heidelberg retinal tomograph. Doc Ophthalmol (in with the
press).

7 Zambarakji H, Butler T, Vernon SA. Assessment of the Heidelberg retina tomograph in the detection of sight threatening diabetic retinopathy. Eye 1999;13:136-44. 